\title{
Atypical Presentation of Complicated Dermoid Cyst of Ovary Mimicking Diverticular Abscess
}

\author{
Prasun Das ${ }^{1}$, Sudipta Basu ${ }^{2}$, Srijak Bhattacharyya ${ }^{3}$, Swadha Priya Basu ${ }^{4}$, \\ Sohini Parveen Sarwala ${ }^{5}$ \\ ${ }^{1,3}$ Post Graduate Trainee, Department of Radio-Diagnosis, Nilratan Sircar Medical College and Hospital, \\ Kolkata, West Bengal, India \\ ${ }^{2}$ Associate Professor, Department of Radio-Diagnosis, Nilratan Sircar Medical College and Hospital, Kolkata, \\ West Bengal, India \\ ${ }^{4}$ Professor and Head, Department of Radio-Diagnosis, Nilratan Sircar Medical College and Hospital, Kolkata, \\ West Bengal, India \\ ${ }^{5}$ Post Graduate Trainee, Department of Pathology, Nilratan Sircar Medical College and Hospital, Kolkata, West \\ Bengal, India
}

Corresponding Author: Swadha Priya Basu

\begin{abstract}
Background: Cystic teratomas comprises $20 \%$ overall among all ovarian neoplasia. They are made of derivatives of at least two of three germ layers. Histologically different dermoid cyst contains only well differentiated embryological elements of ectodermal layer. Diverticulitis of colon mainly affects elderly population having abscess formation causing lower abdominal pain.
\end{abstract}

Case presentation: We report a case of $50 \mathrm{yr}$ old woman presenting with lower abdominal pain. From barium enema, ultrasound and CT we suggested the probability of diverticular abscess. Patient was undergone left salpingooophorectomy with repair of adhered part of sigmoid colon. Post op biopsy shows typical features of dermoid cyst.

Conclusions: We illustrate how a dermoid cyst may be diagnosed as diverticular abscess by imaging. We also showed the rupture of dermoid cyst into sigmoid colon with communication formation. So we the radiologists must be aware of all possible differential diagnosis of lower abdominal pain in elderly female, so that we can help our fellow surgeons to treat properly.

Keywords: Cystic teratoma, Diverticulitis, Post menopausal, Sigmoid Colon

\section{INTRODUCTION}

Teratoma, the most common germ cell tumour of ovary, comprises of $20 \%$ of all ovarian neoplasms. Pathologically they are pluripotent and subdivided into mature, immature and some monodermal varieties. Histologically it includes more than one well differentiated germ cell layers. It has a variety of clinical and radiological presentations as well as many complications involving surrounding structures. Colonic diverticulitis is a complication of diverticulosis that mostly affects elderly patients.

\section{CASE REPORT}

A $50 \mathrm{yr}$ old postmenopausal woman presented in surgical out-patient department of our institution with lower abdominal pain for 6 months with mild alteration of bowel habit for last 1 month and low grade fever for last 15 days. She had no significant weight loss or any clinical signs of blood loss (i.e. pallor). She had mild neutrophilic leukocytosis. She was first suggested by on duty surgical residents to undergo barium enema to rule out any pathology involving the lower alimentary tract. We $1^{\text {st }}$ took consent for all relevant investigations. On barium enema, a large outpouching noted in 
close proximity with anterior wall of opacified sigmoid colon (figure no 2, 3). On plain film we observed a calcific foci in the left side of pelvis (figure no 1). On USG, in left iliac fossa, a well defined hypoechoic area of collection, measuring $4.3 * 3 \mathrm{~cm}$ in size with internal echoes, debris and fine septations without internal vascularity appearing to be adhered to the surrounding gut loop (figure no 4). As the patient was post menopausal, uterus and bilateral ovaries had atrophic changes. On computed tomography, we found eccentric foci of calcification having 208 Hounsfield value within a cystic hypodense structure in left iliac region having air fluid level. Mild enhancement of cyst wall was observed after contrast enhancement. The density within the fluid part of that cystic lesion was same as the oral contrast in the lumen of sigmoid colon. The posterior wall of that lesion seem to have communication with the anterior wall of sigmoid colon (figure no 6, 7).

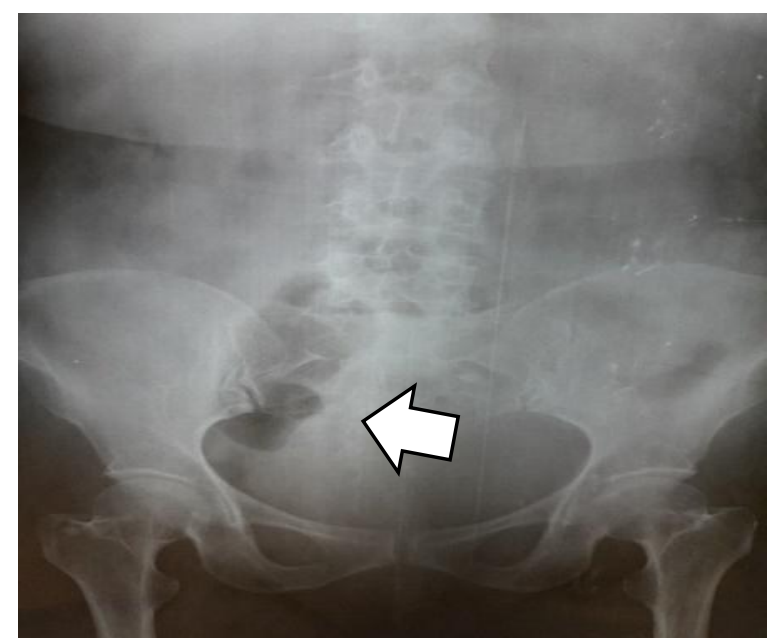

Figure no 1: plain abdominal radiograph showing a calcific foci just in the region of left sacro iliac joint.

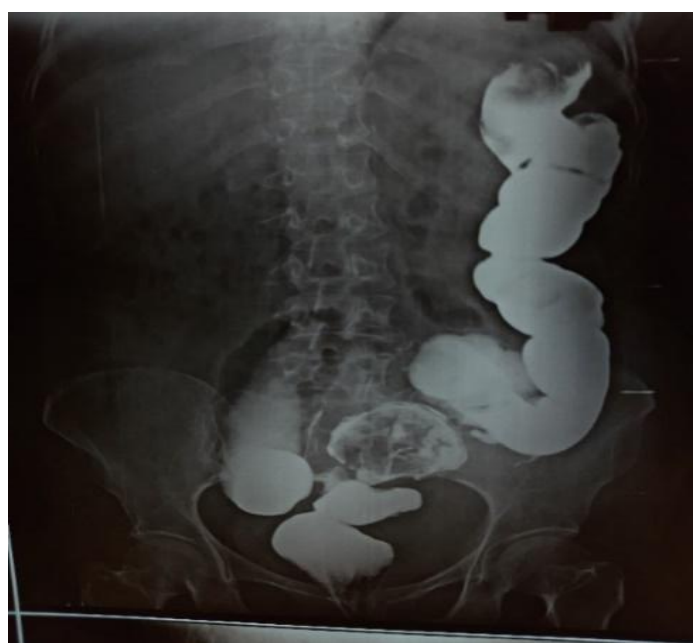

(2)

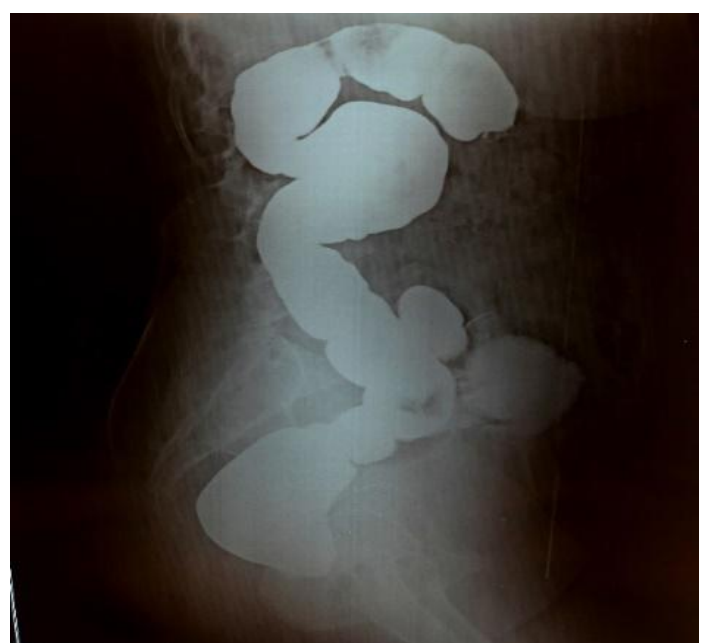

(3)

Figure no 2,3: showing barium enema study demonstrating a radiolucent cystic structure with contrast opacified wall and few internal septations,appeared to be communicated with anterior wall of sigmoid colon

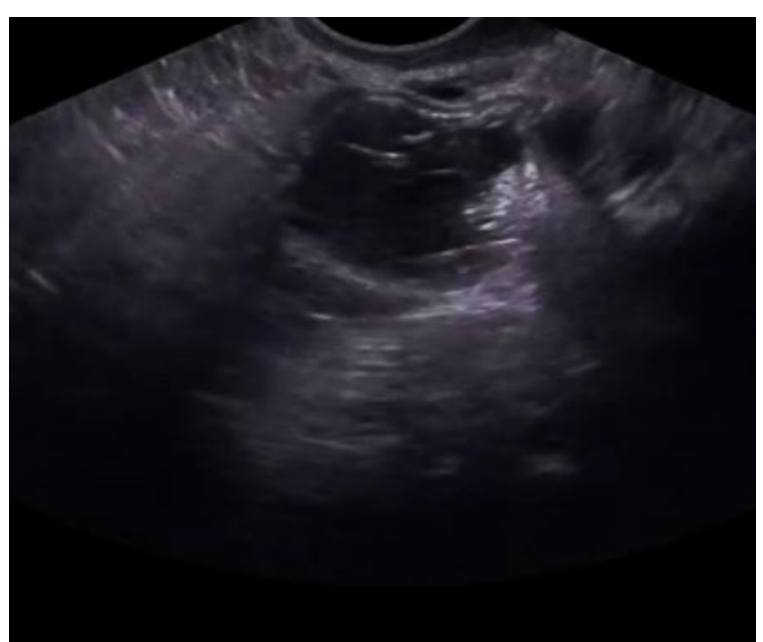

(4)
Figure no 4 shows ultrasound done in left iliac fossa revealing a hypoechoic area of collection with internal calcific material and tiny multiple septations near the gut loops

We suspected that lesion to be diverticular abscess. On operation table the surgeons while removing that cyst found that its posterior wall had adhesion with anterior wall of sigmoid colon and its lumen had communication with the lumen of sigmoid colon. Left sided salpingo oophorectomy was done along with repair of anterior wall of sigmoid colon and the specimen was sent for histo-pathological examination. Grossly it was found to be an 
ovarian mass measuring $4.5 * 3.5 \mathrm{~cm}$ with smooth external surface having cut surface which was filled with sebum like greasy material near the adhered part. Within it there was a Rokitansky nodule with the mucinous internal contents \& cut sections of few hair follicles walled by contained squamous keratinizing epithelium. (Figure no 7)

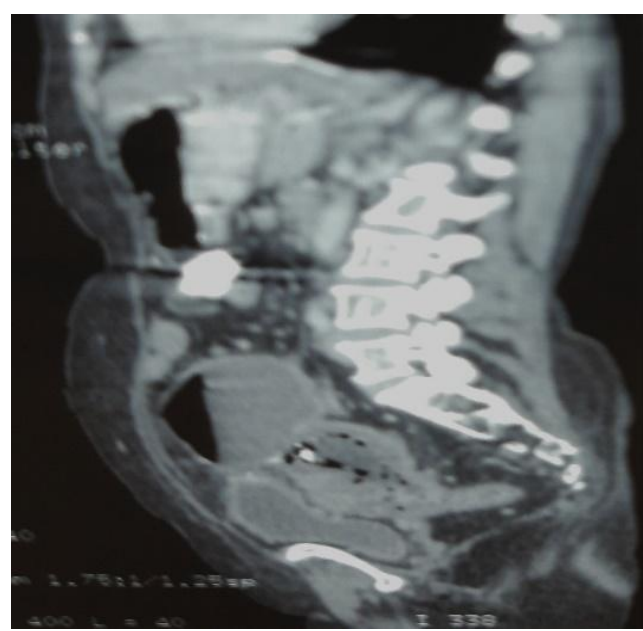

(5)

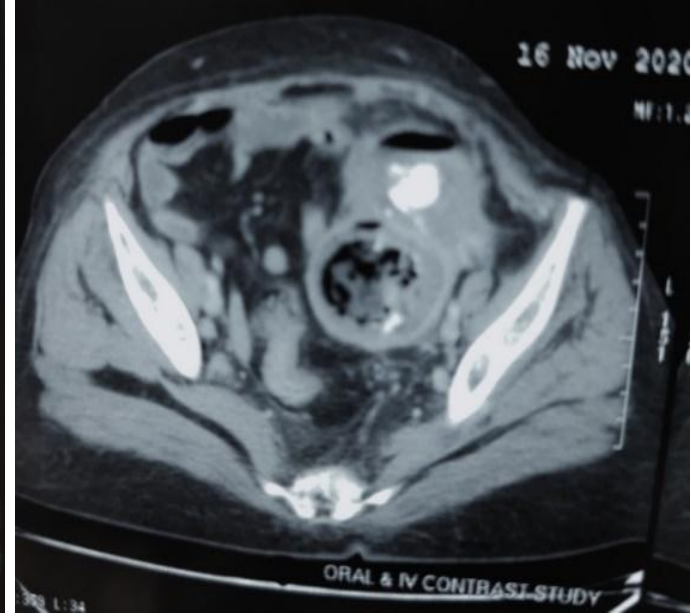

(6)

Figure no 5,6 shows computed tomogram revealing the same cystic structure with isodense regular wall and internal hypo to isodense collection and hyperdense calcification.It appears to be arising from sigmoid colonic wall as shown having gut signatures with multiple dirty air densities.

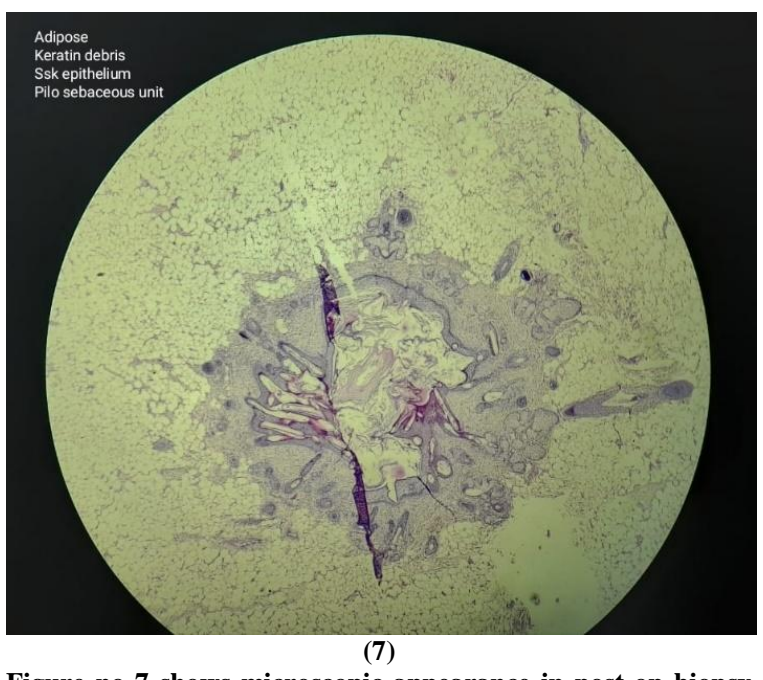

Figure no 7 shows microscopic appearance in post op biopsy revealing adipose tissue, keratin debris, pilosebaceous unit

\section{DISCUSSION}

There are different US observations of teratoma resembling other adnexal masses. Any complication may obscure the imaging findings of ovarian teratomas. ${ }^{(1)}$ Mature cystic teratoma commonly occurs in reproductive age. Usually asymptomatic, symptoms may be caused by torsion or rupture. A Rokitansky protuberance refers to a mural nodule inside which bone, teeth or hair are normally found. ${ }^{(2)}$ Many have mixed echotextures and their image varies based on their composition.

Histologically we see stratified squamous epithelium, keratin debris, pilosebaceous unit (ectoderm); bone, cartilage, adipose (mesoderm) Hemorrhagic cysts, intestinal loops, perforated appendix with appendicolith, ovarian neoplasms with echogenic mural nodules such as lipoleiomyoma and myolipoma may resemble it. ${ }^{(3)}$ Mature cystic teratomas are surgically treated due to the possibility of complications, commonly, torsion (3.5\%$16 \%) .{ }^{(4)}$ Malignant transformation $(1-2 \%)$ is mainly seen in postmenopausal women. Haemorrhage and torsion with hemorrhagic infarction can increase the attenuation of the cyst. The tumor may rupture causing granulomatous peritonitis. (5) Dermoid tumors of extrapelvic organs including rectum, pancreas, kidney, round ligament and testis have been reported. Herniations of the mucosa and portions of the submucosa through the muscularis propria are acquired by the colonic diverticula. Uncomplicated diverticulosis occurs in $75 \%$ of cases. It can be difficult to treat diverticulitis complicated by an abscess, fistula, 
obstruction or perforation (table no 1). ${ }^{(6)}$ The main method of testing patients with possible diverticulitis has historically been contrast enema which has now been replaced by CT scan. ${ }^{(7)}$ Supine and upright abdominal radiographs are normally diagnostic only in the most extreme cases. It is harder to describe the contents of the intramural mass. ${ }^{(8)}$ Though multidetector CT (MDCT) is the primary cross-sectional imaging study for patients with suspected diverticulitis, the first study ordered for nondescript abdominal pain could be ultrasonography (US). The presence of inflammatory changes in the pericolic fat is the characteristic of CT finding of acute diverticulitis. In moderate cases, the attenuation of fat next to the colon is just marginally increased. Fine linear strands, small collections of fluid, and some extraluminal air bubbles can be present. ${ }^{(9)}$

Table no 1 showing modified Hinchey's classification of Diverticulitis

\begin{tabular}{|l|l|}
\hline Stages & Pathology \\
\hline 0 & Mild clinical Diverticulitis \\
\hline Ia & Confirmed pericolic inflammation/phlegmon \\
\hline Ib & Pericolic or mesocolic abscess \\
\hline II & Pelvic,distant intra-abdominal /retroperitoneal abscess \\
\hline III & Generalised purulent peritonitis \\
\hline IV & Generalised fecal peritonitis \\
\hline
\end{tabular}

Our case is of an atypical complication of complicated ovarian dermoid cyst presenting with chief complaint of lower abdominal pain. On barium enema, Ultrasound and computed tomography, we suspected it to be a large diverticular abscess from sigmoid colon. On post op finding, that mass was found to be dermoid communicating with sigmoid colon. The calcific foci on plain abdominal radiograph and non enhanced computed tomogram found to be Rokitansky nodule with tooth.

Differential diagnosis list:

1. Diverticulitis with abscess formation

2. Cystic degeneration of uterine fibroid

3. Cystic colonic carcinoma

4. Left sided psoas abscess

\section{CONCLUSIONS}

One rare differential diagnosis of diverticular abscess include complicated dermoid cyst if situated in the curvature of sigmoid colon. So only different imaging modalities can not surely narrow down to one single diagnosis, specially in post menopausal status as in our case. We have to follow up upto post op biopsy report for correct diagnostic management. Mature cystic teratoma has wide spectrum of appearances in different imaging modalities. It is also a good mimic of many pathologies in lower abdomen with almost comparable clinical features. We the radiologists must be aware of various complications of atypically presented dermoid cyst, so that we can help fellow surgeons to correctly diagnose and treat them accordingly.

\section{Authors Contribution}

Dr. Prasun Das: Acquisition of data, Drafting of manuscript

Dr. Sudipta Basu: Study concept \& design, Analysis of data, Critical Revision

Dr. Swadha Priya Basu: Supervisor

Dr. Srijak Bhattacharyya: Acquisition of data

Dr. Sohini Parveen Sarwala: Acquisition of data

\section{Acknowledgement: None}

Conflict of Interest: None

\section{Source of Funding: None}

\section{Declaration of Patient Consent}

Appropriate patient consent was taken prior to publication in the journal.

\section{REFERENCES}

1. Shaaban AM et al: Ovarian malignant germ cell tumors: cellular classification and clinical and imaging features. Radiographics. 34(3):777-801, 2014

2. Rim SY et al: Malignant transformation of ovarian mature cystic teratoma. Int $\mathrm{J}$ Gynecol Cancer. 16(1):140-4, 2006 
3. Kim HC et al: Fluid-fluid levels in ovarian teratomas. Abdom Imaging. 27(1):100-5, 2002

4. Outwater EK et al: Ovarian teratomas: tumor types and imaging characteristics. Radiographics. 21(2):475-90, 2001

5. Jeong YY et al: Imaging evaluation of ovarian masses. Radiographics. 20(5):144570, 2000

6. Rafferty J, Shellito P, Hyman NH, et al: Practice parameters for sigmoid diverticulitis. Dis ColonRectum 49:939944, 2006.

7. Vijayaraghavan SB: High-resolution sonographic spectrum of diverticulosis, diverticulitis, and their complications. J Ultrasound Med 25:75-85, 2006.
8. Rotert H, Nöldge G, Encke J, et al: The value of $\mathrm{CT}$ for the diagnosis of acute diverticulitis.Radiologe 43:51-58, 2003.

9. Rotert H, Nöldge G, Encke J, et al: The value of CT for the diagnosis of acute diverticulitis.Radiologe 43:51-58, 2003.

10. Chintapalli KN, Chopra S, Ghiatas AA, et al: Diverticulitis versus colon cancer: Differentiation with helical CT findings. Radiology 210:429-435, 1999.

How to cite this article: Das P, Basu S, Bhattacharyya $S$ et.al. Atypical presentation of complicated dermoid cyst of ovary mimicking diverticular abscess. International Journal of Research and Review. 2021; 8(6): 30-34. DOI: https://doi.org/10.52403/ijrr.20210605 